\title{
PRINCIPIO DE CONTINUIDAD EN EL RÉGIMEN LABORAL DE LOS DOCENTES DE INSTITUCIONES EDUCATIVAS PARTICULARES DEL PERÚ
}

\section{PRINCIPLE OF CONTINUITY IN THE LABOR REGIME OF THE TEACHERS OF PARTICULAR EDUCATIONAL INSTITUTIONS OF PERU}

\author{
Claudia Sofia Valdeiglesias Pacheco \\ Docente \\ Universidad Andina del Cusco \\ sofiapa86@hotmail.com \\ Perú
}

\section{SUMARIO}

- Regímenes laborales en el Perú

- El régimen laboral de la actividad privada

- Contrato laboral de docentes de instituciones educativas privadas

- Principio de continuidad en el empleo

- Conclusiones

\section{RESUMEN}

En el sector educativo privado, conforme lo establece la Ley $\mathrm{N}^{\circ} 28044$, Ley General de Educación y el Decreto Legislativo N882, Ley de Promoción en la Inversión en Educación; los docentes de las instituciones educativas particulares se encuentran comprendidos en el régimen laboral de la actividad privada. Las relaciones laborales en instituciones educativas particulares tienen peculiaridades debido a los contratos de docentes a tiempo completo, mediante contratos modales.

Si bien es cierto, actualmente SUNAFIL viene abordando el tema con autoridades de las instituciones educativas privadas, el presente artículo busca determinar que aún existe vulneración del principio constitucional de continuidad en el empleo, específicamente en los contratos que suscriben los docentes del régimen laboral de instituciones educativas privadas, pues se considera que la continuidad laboral del trabajador se ve afectada, en virtud a que se elude y reduce la CTS, gratificaciones legales, y se elimina el descanso vacacional físico; en consecuencia se puede concluir que existe una modalidad contractual fraudulenta.
En este entender el docente de una institución educativa privada tendría derecho a la protección contra el despido arbitrario, mediante la continuidad en el empleo y la estabilidad laboral siempre que desarrolle labor permanente en su función educativa.

\begin{abstract}
In the private educational sector, as established by Law No. 28044, General Education Law and Legislative Decree No. 882, Law of Promotion in Investment in Education; Teachers of private educational institutions are included in the labor regime of private activity. Labor relations in particular educational institutions have peculiarities due to full-time teacher contracts, through modal contracts.
\end{abstract}

While it is true, SUNAFIL is currently addressing the issue with authorities of private educational institutions, this article seeks to determine that there is still a violation of the constitutional principle of continuity in employment, specifically in the contracts signed by teachers in the labor regime of institutions private educational, since it is considered that the worker's labor continuity is affected, by virtue of avoiding and reducing the CTS, legal bonuses, and eliminating the physical vacation rest; consequently, it can be concluded that there is a fraudulent contractual modality.

In this understanding the teacher of a private educational institution would have the right to protection against arbitrary dismissal, through continuity in employment and job stability 
provided that he develops permanent work in his educational role.

\section{PALABRAS CLAVE}

Continuidad laboral, contrato laboral, docentes, instituciones educativas privadas.

\section{KEYWORDS}

Labor continuity, employment contract, teachers, private educational institutions.

\section{REGÍMENES LABORALES EN EL PERÚ}

A manera de antecedentes, que la Constitución Política del Perú del 1979 establecía un sistema único que homologaba las remuneraciones, bonificaciones y pensiones de los servidores del Estado. Se puede afirmar que durante su vigencia existía un sistema único; sin embargo, dicha norma no se plasmó realmente. Posteriormente en virtud a la entrada en vigencia de la Constitución de 1993, la norma homologatoria fue eliminada, lo que generó dispersión no solo en la legislación laboral, sino en la estructuración remunerativa.

El Informe Final de la Comisión Multisectorial encargada de estudiar la situación del personal de la Administración Pública Central, señala que en la década de los ochenta fueron unificados dentro del régimen de la actividad privada todos los trabajadores de las empresas del Estado; en tanto, que en la década de los noventa, en un proceso de reforma que quedó trunco, comenzaron a migrar hacia el régimen laboral privado una serie de entidades e instituciones públicas, generándose una multiplicidad de organismos y sistemas de pagos al personal, una gran variedad de conceptos remuneratorios y no remuneratorios y de montos, al punto que no había dos ministros, viceministros o secretarios generales que ganaran igual cantidad.

A partir de los noventa, los servidores públicos empezaron a ser contratados bajo dos tipos de regímenes laborales distintos: el regulado por el Decreto Legislativo $\mathrm{N}^{\circ} 276$ o por el Decreto Legislativo $\mathrm{N}^{\circ} 728$.

Luego, a propósito del Informe final de la Comisión Multisectorial encargada de estudiar la situación del personal de la Administración
Pública Central, se aprueba un nuevo régimen laboral, regulado por el Decreto Legislativo $\mathrm{N}^{\circ}$ 1057, denominado Contratación Administrativa de Servicios (CAS), que vendría a corregir un fenómeno que se empezó a desarrollar dentro del sector público a finales de los noventa con la contratación de servidores bajo la modalidad de Servicios No Personales (SNP). (Cruzado Medina, 2011)

El tema de la legislación laboral en el Perú, durante los últimos 50 años, se caracteriza por ser un periodo en el cual surgieron varios regímenes por actividad y tipo de trabajadores. En la actividad pública, entre regímenes laborales generales y especiales existen 15 (diplomáticos, fiscales, gerentes públicos, militares y policías, CAS y otros). En tanto, para la actividad privada, son 24 los regímenes laborales especiales (agrarios, personal de confianza, exportación no tradicional, microempresa, entre otros).

\section{EL RÉGIMEN LABORAL DE LA ACTIVIDAD PRIVADA}

Los trabajadores que pertenecen al régimen de la actividad privada tienen derecho de recibir las facultades concedidas y prescritas en la ley, tales como: derecho a gozar de vacaciones anuales por 30 días, a percibir dos gratificaciones anuales equivalentes a un sueldo cada una en los meses de julio y diciembre, a la compensación por tiempo de servicios (CTS) en un monto algo superior a una remuneración por año y a una indemnización por despido arbitrario tarifada en un sueldo y medio por periodo anual laborado si el contrato es pactado a plazo indefinido (Serkovic, 2015).

El régimen laboral privado coexiste con regímenes especiales de carácter promocional, algunos basados en las características específicas de la labor por realizarse, como el caso de los trabajadores agrarios o con reservas de los dependientes de empresas dedicadas a la exportación no tradicional; otros sustentados en el tamaño de la empresa en función de la cuantía de sus ventas, como son los de las micro y pequeñas empresas. Este régimen se encuentra regulado por el Decreto Legislativo $\mathrm{N}^{\circ} 728$, Ley de Productividad y Competitividad Laboral, orientado a constituir un régimen de igualdad de oportunidades de empleo que asegure a todos los ciudadanos el acceso a 
una ocupación útil que los proteja contra el desempleo y el subempleo en cualquiera de sus manifestaciones.

\section{Régimen laboral de las instituciones educativas privadas en el Perú}

La Constitución Política del Perú, en el ámbito de la educación, genera bastantes conceptos que son necesarios esclarecer y resaltar en la presente investigación.

En el Perú se ofrece la educación en el sector público y privado; para efectos del presente artículo, se revisará puntualmente la oferta privada en el rubro educativo, por ello es necesario citar lo establecido en el art. 15 de la Constitución Política del Perú, Régimen del profesorado:

El profesorado, en la enseñanza oficial es considerado carrera pública. La ley establece los requisitos para desempeñarse como director o profesor de un centro educativo, así como sus derechos y obligaciones. El Estado y la sociedad procuran su evaluación, capacitación, profesionalización y promoción permanentes. El educando tiene derecho a una formación que respete su identidad, así como al buen trato psicológico y físico. Toda persona, natural o jurídica, tiene el derecho de promover y conducir instituciones educativas y el de transferir la propiedad de éstas, conforme a ley.

\section{Antecedentes y normativa del régimen laboral de las instituciones educativas privadas en el Perú.}

A nivel constitucional, se debe recordar la Ley $\mathrm{N}^{\circ} 24029$, Ley del Profesorado, que estuvo vigente desde el 21 de mayo de 1990 hasta el 25 de noviembre de 2012, modificada por la Ley $\mathrm{N}^{\circ} 25212$, donde se establecía en su título IV "Del régimen del profesor particular", artículos 61 al 63

Artículo 61.- El servicio particular comprende a los profesores que trabajan en el área de la docencia en centros y programas educativos de gestión no estatal. $\left(^{*}\right)$

(*)Artículo modificado por la Primera Disposición Final del Decreto Ley $N^{\circ} 26011$, publicado el 27 12-92, cuyo texto es el siguiente:
Artículo 61.- El servicio particular comprende a los profesores que trabajan en el área de la docencia en centros y programas educativos de régimen laboral de la actividad privada, así éstos reciban recursos provenientes del Estado.

Artículo 62.- El profesorado del servicio particular está sujeto al régimen laboral de la actividad privada.

$\mathrm{Su}$ jornada laboral tiene el límite máximo señalado en el Artículo 19 de la presente Ley. (*)

(*) Artículo modificado por el Artículo 1 de la Ley $\mathrm{N}^{\circ}$ 25212, publicada el 20-05-90, cuyo texto es el siguiente:

Artículo 62.- El profesorado del servicio particular está sujeto al régimen laboral de la actividad privada.

La jornada laboral se sujeta a lo establecido en el Artículo 18 de la presente ley.

Las remuneraciones de los docentes con título pedagógico, no serán menores a las que perciben los profesores al servicio del Estado. Por cada incremento de las pensiones de enseñanza de cada centro educativo serán incrementadas las remuneraciones de sus respectivos docentes.

Artículo 63.- El sistema de evaluación del profesorado particular se determina en el Reglamento Interno del Centro Educativo considerando los aspectos básicos señalados en el Artículo $\mathrm{N}^{\mathrm{o}} 38$.

La hoja anual de evaluación será enviada al Órgano Departamental o Zonal del Ministerio de Educación para su archivo. Este documento acredita los servicios y el consiguiente derecho de los profesores a las prestaciones de la Seguridad Social.

Dicho antecedente fue reglamentado a través del Decreto Supremo N019-90-ED, artículos 260 al 267, de sus artículos se puede concluir que los profesores de las instituciones privadas fueron incluidos en el régimen laboral privado sin dejar de precisar que fueron homologados en algunos derechos con el régimen público, tales como: la estabilidad laboral en la plaza, jornada ordinaria de horas pedagógicas, las horas extras.

Las instituciones educativas privadas en el Perú conforme lo ha establecido la Ley N²8044, 
Ley general de Educación en su artículo 61 establece:

Artículo $61^{\circ}$.- Régimen laboral del Profesor en la Educación Privada. El profesor que trabaja en instituciones educativas privadas se rige por lo establecido en el régimen laboral de la actividad privada. Puede incorporarse en la carrera pública magisterial si ingresa al servicio del Estado, previo cumplimiento de los requisitos de ley.

El Decreto Legislativo $\mathrm{N}^{\circ} 882$, Ley de Promoción en la Inversión en la Educación en su artículo $6^{\circ}$ establece: "Artículo $6^{\circ}$.El personal docente y los trabajadores administrativos de las Instituciones Educativas Particulares, bajo relación de dependencia, se rigen exclusivamente por las normas del régimen laboral de la actividad privada."

Con las normas antes citadas, queda claro que el régimen laboral al cual están sujetos los docentes de las instituciones educativas privadas es el Régimen Laboral Privado; se rigen por lo establecido en el Decreto Legislativo $\mathrm{N}^{\circ} 728$, Ley de Productividad y Competitividad Laboral, aprobado por el Decreto Supremo 003-97-TR (LPCL).

Actualmente, las instituciones educativas privadas, estando bajo el régimen de la actividad privada, mantienen una relación laboral con sus trabajadores bajo contratos sujetos a modalidad, al tener contratos por periodos comprendidos de marzo a diciembre, un lapso de 10 meses; desde el inicio del año escolar (marzo) hasta la finalización del año escolar (diciembre).

Con dichas contrataciones se evidencia que durante los meses de enero y febrero aparentemente no existiría una relación laboral, pese a ser nuevamente contratado durante el mes de marzo de año lectivo siguiente.

\section{Regulación de la actividad laboral de docentes en el sector público y privado}

Por otro lado, la Ley de Reforma Magisterial (LRM) no beneficia a los docentes que enseñan en las instituciones privadas. Esta situación ha generado que se marquen varias diferencias laborales entre los docentes del sector público y privado.

El Presidente Ejecutivo de CORPAIDOS, Alfredo Aguilar (Silvera Quispe, 2013), explica que la LRM fue diseñada "específicamente" para el sector público. "Los colegios privados se rigen bajo el DL N ${ }^{\circ} 728$ y tienen autonomía pedagógica, económica y de régimen laboral", sostiene.

A nivel nacional la cifra alcanza los $163 \mathrm{mil}$ 853 profesionales. A decir de Aguilar, una de las principales diferencias entre los regímenes laborales estatal y privado es la estabilidad laboral.

"Un maestro del sector privado nunca podrá nombrarse, solo le renovarán el contrato", asegura. Mientras que los educadores de colegios públicos sí tienen la opción de acceder a una plaza fija (previo concurso). La LRM tampoco incluía a los docentes contratados. No obstante, esta medida fue modificada para que los "educadores temporales" puedan acceder a una plaza de nombramiento a partir del 2014

"Lo mismo deberían hacer con los docentes de colegios privados", Estamos frente a una diferenciación que puede interpretarse como una marginación laboral. Cada año, al menos 100 maestros se quejan de los atropellos en los colegios privados, sobre todo por temas remunerativos.

Lo ideal sería que todos los profesores en todos sus niveles inicial, primaria y secundaria sean en futuro reunidos en un solo régimen laboral especial, dado el carácter de sus funciones.

Mientras en la LRM se ofrece ocho niveles de salarios que van desde los dos mil soles (Nivel I) hasta los S/. 5,390 (Nivel VII); en los colegios particulares varía de acuerdo al plantel. Va desde el salario mínimo hasta los $\mathrm{S} / .4$ mil aproximadamente.

No solo se cuestiona el tema remunerativo. Los maestros de un colegio particular, observando la forma de contratación, no llegan a gozar de vacaciones remuneradas. Trabajan diez meses (de marzo a diciembre) y termina el vínculo laboral. En ese momento les pagan el equivalente a un mes de trabajo, por concepto de vacaciones.

Aguilar detalla que este sistema es utilizado en la mayoría de colegios particulares. En la LRM los maestros gozan de dos meses (60 días) de vacaciones al año. 


\section{CONTRATO LABORAL DE DOCENTES DE INSTITUCIONES EDUCATIVAS PRIVADAS}

En el sector educativo privado, conforme establece la Ley $\mathrm{N}^{\circ} 28044$, Ley General de Educación en su artículo $61^{\circ}$, el Decreto Legislativo $\mathrm{N}^{\circ} 882$, Ley de Promoción en la Inversión en Educación en su artículo $6^{\circ}$; los docentes de las instituciones educativas privadas se encuentran regulados en el régimen laboral de la actividad privada, es decir, según lo establecido en el Texto Único Ordenado del Decreto Legislativo N²728, Ley de Productividad y Competitividad Laboral, que dispone que la contratación laboral debe constar por escrito debiendo consignarse de forma expresa su duración y las causas objetivas determinantes de la contratación. No es suficiente invocar en el contrato de trabajo sujeto a modalidad la causal en que se sustenta, sino que dicha causa debe realmente haberse configurado para que proceda la contratación temporal; en consecuencia, si no se evidencia una causa temporal de contratación, estaremos ante un contrato a plazo indeterminado.

Por su parte, el Tribunal Constitucional ha establecido respecto a la continuidad en el empleo, en la sentencia recaída en el Exp. N¹397-2001-AA/TC (Continuidad y causalidad, 2001), fundamento tres: “el régimen laboral peruano se sustenta entre otros criterios, en el llamado principio de causalidad, en virtud del cual la duración del vínculo laboral debe ser garantizada mientras subsista la fuente que le dio origen. En tal sentido hay una preferencia por la contratación laboral por tiempo indefino".

En virtud a lo expuesto, se debe precisar que los docentes de instituciones educativas particulares vienen siendo contratados bajo contratos modales desde el inicio del año escolar, en el mes de marzo, hasta finales del mes de diciembre; es decir, por un periodo de diez meses, en consecuencia, el docente no tiene vínculo laboral durante los meses de enero y febrero.

En el caso que se viene tratando, resulta evidente que el empleador limita el derecho al trabajo, el cual se encuentra regulado en los artículos $22^{\circ}$ y $23^{\circ}$ de la Constitución Política del Perú, ya que la temporalidad indeterminada de los contratos laborales busca armonizar la legislación ordinaria con las disposiciones de la Constitución, en cuanto a que el trabajo sea la base del bienestar social y un medio de realización de la persona (Gonzales, 2013).

Es así, que la continuidad laboral del trabajador se ve afectada, pues se elude y reduce la CTS, gratificaciones legales, y se elimina el descanso vacacional físico. En este entender, se evidencia la existencia de una modalidad contractual fraudulenta.

El artículo $77^{\circ}$ literal d) del Texto Único Ordenado del Decreto Legislativo N728, Ley de Productividad y Competitividad Laboral, aprobado por Decreto Supremo N003-97-TR, establece que los contratos de trabajo sujetos a modalidad se consideran como de duración indeterminada, cuando el trabajador demuestre la existencia de simulación o fraude a las normas establecidas. Aplicando el principio de primacía de la realidad, se observa que los docentes cumplen una actividad permanente dentro de las instituciones educativas, por lo que no existiría causa alguna de temporalidad para su contratación modal. Por ello se impone vigilar la eficacia del principio de continuidad en el empleo del docente de las instituciones privadas, considerando que al superar el periodo de prueba tiene derecho a la continuidad laboral y a la protección contra el despido arbitrario, siempre que su jornada laboral sea de 4 horas diarias a más.

Frente a la falta de comprensión por parte de las instituciones educativas privadas del principio a la continuidad laboral, podemos citar el Exp. N00231-2017-0-1501-JR-LA03 Sala Laboral Permanente de Huancayo, que establece que:

El contrato modal suscrito no ha justificado de modo objetivo el supuesto incremento de la actividad de la institución educativa privada en el servicio de educación inicial que prestaba, por lo que se estableció que en realidad lo que unió a las partes fue un contrato de trabajo a plazo indeterminado, por lo que la demandante tiene derecho a la protección frente al despido arbitrario. (Reposición, 2017)

En este orden de ideas, se puede verificar la realidad educativa nacional en el tratamiento laboral de los docentes de las instituciones educativas privadas, por lo que es importante la participación de la SUNAFIL en cuanto a las acciones preventivas y el Poder Judicial 
en atender los pedidos de los afectados, ello impone vigilar la eficacia del principio de continuidad en el empleo de los docentes por los órganos supervisores del Estado.

\section{PRINCIPIO DE CONTINUIDAD EN EL EMPLEO}

Este es también denominado como de estabilidad y permanencia, representa la garantía del trabajador de desarrollar su actividad laboral de manera continua e indefinida $\mathrm{o}$, en su defecto, por el espacio de tiempo que exige las actividades para las que fue contratado. El trabajador desarrolla su actividad laboral de manera continua durante la vigencia del contrato de trabajo. De esta manera los trabajadores podrán tener la tranquilidad de que su vinculación laboral se sujetará al espacio de tiempo necesario para el desarrollo de las actividades para las que fueron contratados (Gonzales, 2013).

La naturaleza jurídica del principio se basa en que normalmente el trabajo es la principal fuente de ingreso económico, por lo que el contrato debe considerarse lo más extenso posible, para así beneficiar al trabajador en la consolidación de situaciones jurídicas relacionadas con el trabajo.

El contrato de trabajo constituye un "negocio jurídico bilateral que tiene por finalidad la creación de una relación jurídico - laboral constituida por el cambio continuado entre una prestación de trabajo dependiente y por cuenta ajena y una prestación salarial"(Montoya Melgar, 2014).

De esta manera, la relación laboral nace para prolongarse en el tiempo por cuanto la actividad productiva del empleador ha sido pensada para desarrollarse en forma indefinida y precisamente, para la obtención de resultados es que necesita la prestación de servicios del trabajador en régimen de subordinación. La estrecha relación existente entre la contratación laboral y el desarrollo de las actividades productivas del empleador resulta evidente:

si una empresa ha sido creada para realizar sus actividades y cumplir sus objetivos en un plazo indeterminado, resulta lógico y por ende razonable que los trabajadores, que tienen a su cargo cumplir ese objetivo, permanezcan en sus puestos de trabajo, tanto tiempo como ello sea posible y naturalmente, a decisión del trabajador, que es libre de renunciar (...). (Morales Corrales, 2009)

En consecuencia, la relación laboral está normada por el Principio de Continuidad que considera al contrato de trabajo como uno de duración indefinida, resistente a las diversas circunstancias que, en el desarrollo de la relación laboral, puedan alterar ese carácter de modo que el trabajador puede trabajar mientras quiera, mientras pueda y mientras exista la fuente de trabajo. Este principio "se encuentra íntimamente vinculado a la vitalidad y resistencia de la relación laboral, a pesar que determinadas circunstancias puedan aparecer como razón o motivo de su terminación" de forma que la extinción del contrato de trabajo constituye una situación excepcional predeterminada por ley.

El Art. $22^{\circ}$ de la Constitución recoge, implícitamente, el Principio de Continuidad por cuanto el derecho al trabajo que reconoce, en su aspecto individual, se concreta en el igual derecho de todos a un determinado puesto de trabajo y en el derecho a la continuidad o estabilidad del trabajador en su puesto de empleo de modo que no sea despedido si no existe causa justa.

Como puede verse, el Principio de Continuidad garantiza al trabajador que su contratación, por regla general, deberá efectuarse a plazo indeterminado y que permanecerá inalterable en su puesto de empleo mientras no exista alguna causa de extinción de la relación laboral y la fuente de la actividad productiva del empleador se mantenga.

Al respecto, el Tribunal Constitucional ha manifestado que "en el régimen laboral peruano, el principio de continuidad opera como un límite a la contratación laboral por tiempo determinado. Por ello, este Tribunal, en la Sentencia recaída en el Expediente $\mathrm{N}^{\mathrm{o}}$ 1874-2002-AA/TC, precisó que hay una preferencia por la contratación laboral por tiempo indefinido respecto de la de duración determinada, la que tiene carácter excepcional y procede únicamente cuando las labores que se van a prestar (objeto del contrato) son de naturaleza temporal o accidental. 
En resumen, este principio de Continuidad o "permanencia" parte de la premisa de que el contrato de trabajo es un contrato de tracto sucesivo, es decir, es un vínculo de duración prolongada en el tiempo entre empleador y trabajador, el cual tiene resistencia en su duración. Se manifiesta de la siguiente manera:

- La ley laboral privilegia la existencia de relaciones laborales a tiempo indefinido.

- Presunción de contrato a plazo indefinido.

- Amplitud para admitir interrupciones (suspensiones) sin que se produzca la extinción.

- Reglamentación por excepción de los contratos a plazo fijo (modales).

- Restricción en materia de modificaciones unilaterales a la relación laboral.

- El ius variandi tiene limitaciones.

- Resistencia a la terminación incausada o sin justa causa (estabilidad).

Resulta importante precisar que el derecho laboral garantiza la continuidad de la relación laboral ante cambios en la misma. Ello se debe a que, generalmente, el trabajo es la única fuente de ingresos y medio de subsistencia del trabajador y su familia; pero también a que, en la relación laboral, el empleador puede ser sustituido en el cumplimiento de sus obligaciones sin afectar la relación, lo que no pasa con el trabajador.

\section{CONCLUSIONES}

El principio constitucional de continuidad implica el desarrollo incesante de la relación laboral durante la vigencia del contrato de trabajo, salvo hechos ajenos a la contratación (suspensiones por falta grave, despidos etc.) observando que en el derecho laboral se tiene preferencia por la contratación a plazo indefinido frente a la contratación de carácter temporal.

En el caso de los docentes de instituciones educativas privadas, se evidencia la vulneración del principio constitucional de continuidad en el empleo, en razón de que este tipo de trabajadores cuentan indebidamente con contratos modales a plazo determinado, sin considerar el carácter permanente de sus funciones, al ser sus contratos de carácter temporal de marzo a diciembre de cada año.

En consecuencia, el docente de una institución educativa privada tiene derecho a la protección contra el despido arbitrario, mediante la continuidad en el empleo y la estabilidad laboral, siempre que desarrolle una función de carácter permanente en la labor educativa.

\section{FUENTES DE INFORMACIÓN}

\section{Fuentes bibliográficas}

Álvaro, G. R. (2013). Modalidades de contratación laboral. Lima, Perú: Gaceta Jurídica.

Arce, E. (2006). Estabilidad Laboral y Contratos temporales. Lima, Perú: Departamento Académico de Derecho PUCP.

Corrales, R. (junio de 2017). "Régimen Laboral de los Profesores Particulares". Revista Soluciones Laborales, 110-116.

De Los Heros, A. (2009). Los contratos de trabajo de duración determinada regla o excepción. Lima, Perú: Grijley.

Gonzales, L. (2013). Modalidades de Contratación Laboral. Lima, Perú: El Buho.

Neves, J. (2016). Comentarios a la Ley de Productividad y Competitividad Laboral. Lima, Perú: EL Búho.

Castilli, J. (2015). Compendio de Derecho Laboral Peruano. Lima, Perú: Tinco SA.

Palomeque, M. (2008). Derecho del Trabajo. Madrid -España: Centro de Estudios Ramón Areces.

Álvaro, G. R. (2013). Modalidades de contratación laboral. Lima: Gaceta Jurídica S.A.

\section{Fuentes electrónicas}

Paredes, J. (s.f.). www.pj.gob.pe. Recuperado el 26 de Agosto de 2017, de https://www.pj.gob.pe/wps/wcm/connect/ dc3466804b4149d688bb89501dddbb53/CS 
Continuidad y causalidad, 1397 (Tribunal Constitucional - Acción de Amparo 2001). corporacionhiramservicioslegales. (s.f.). corporacionhiramservicioslegales. Recuperado el 24 de agosto de 2017, de https://corporacionhiramservicioslegales. blogspot.pe/2012/10/principio-decontinuidadlaboral-y.html

Concha, C. R. (2014). Análisis de la Estabilidad Laboral de los trabajadores de confianza según el Tribunal Constitucional. Lima Perú. Pontificia Universidad Católica del Perú. Recuperado del sitio de internet: http://tesis.pucp.edu.pe/ repositorio/bitstream/handle/123456789/5244/ CONCHA VALENCIA CARLOS ANALISIS_ESTABILIDAD.pdr? sequence $=\overline{1}$
Corte Suprema de Justicia - Sala Constitucional y Social Transitoria. Expediente: 0021822005. Recuperado del sitio de internet: https:// vlex.com.pe/vid/-472618610

Ginebra, O. I. (2005). Conferencia Internacional del Trabajo, 95.a. Obtenido de La relación de trabajo: http://www.ilo.org/public/spanish/ standards/relm/ilc/ilc95/pdf/rep-v1.pdf 